\title{
FRP-to-Concrete Joint Assemblies Anchored with Multiple FRP Anchors: Experimental Investigation
}

\author{
H.W. Zhang \& S.T. Smith (stsmith@hku.hk) \\ Department of Civil Engineering, The University of Hong Kong, China
}

\begin{abstract}
Higher strains can be developed in fibre-reinforced polymer (FRP) composites which are bonded to the surfaces of concrete members if the FRP is anchored. Anchors made from FRP (also known as FRP spike anchors but herein referred to as FRP anchors) are a promising type of anchorage as they can be applied to a variety of different shaped structural elements and they have been shown to be effective in enhancing the strain capacity of externally bonded FRP. Limited research, however, has been conducted on understanding and quantifying the strength and behaviour of such anchors in isolation and research to date has been on mainly single anchors. A series of tests is therefore reported in this paper on FRP-to-concrete joints anchored with two FRP anchors with the main test variable being the relative position of the anchors. Displacement controlled tests have enabled the complete load-slip responses of the joints to be captured which in turn provides valuable insights to be gained in understanding the behaviour of the anchored joint over the complete loading range. The tests reported in this paper advance our understanding of FRP anchor groups in anchoring externally bonded FRP strengthening systems.
\end{abstract}

\section{INTRODUCTION}

Numerous studies have proven the effectiveness of strengthening concrete with externally bonded fibrereinforced polymer (FRP) composites (e.g. Hollaway and Teng 2008). Numerous studies have also shown the FRP to debond from the concrete at strains substantially lower than the rupture strain of the FRP (Hollaway and Teng 2008). Recent studies have shown the effectiveness of the externally bonded FRP to be increased upon the addition of anchorage (Smith 2009, Zhang et al. 2010). Anchors made from FRP (herein FRP anchors) have been proven to be a most effective form of anchorage as tests have shown the strength of FRP-to-concrete joints to be increased up to $80 \%$ by the introduction of a single FRP anchor (Smith 2009). The bulk of tests conducted to date have, however, been confined to FRP-to-concrete joints with a single FRP anchor. In reality, multiple anchors may need to be installed to the strengthening system and hence our current limit of knowledge needs to be expanded.

This paper reports the results of a series of tests on FRP-to-concrete joints which have been anchored with two anchors. The main test variable is the relative position of the two anchors. These tests reported herein form part of a much larger testing program being conducted at The University of Hong Kong on the characterisation of FRP anchors.

\section{EXPERIMENTAL SET-UP AND DETAILS}

The single shear test set-up utilised in the experimental programme is shown in Figure 1. The main test variables of number of anchors and relative anchor positions are shown in Figure 2. In addition, three control tests were conducted on unanchored FRP-to-concrete joints (not shown in Figure 2) possessing the same concrete and FRP plate geometrical properties as the anchored joints tests.

All FRP plates were formed from three layers of carbon fibre sheet in a wet lay-up procedure $(0.131$ $\mathrm{mm}$ nominal carbon fibre sheet thickness) and a 40 $\mathrm{mm}$ unbonded zone was maintained at the loaded free end of the concrete prism. The impregnated carbon FRP anchors used in this study, which were formed by hand in the laboratory from rolling 200 mm wide carbon fibre sheets (i.e. same sheet used for plate and anchors), are fully described in Zhang et al. (2010). All anchors were embedded to a constant depth of $40 \mathrm{~mm}$ and the anchor fan component was oriented to the direction of load as shown in Figure 1. Additional details of the anchors are provided in Figure 3 with selected photographs of the installation of the FRP anchor and FRP plating in Figure 4.

The concrete cube compressive strength of the unanchored control joints was $50.3 \mathrm{MPa}$ and that of all the anchored joints was $51.9 \mathrm{MPa}$. The FRP me- 
chanical properties, derived from tests on flat coupons, were elongation $=15,172 \mu \varepsilon$ (standard deviation, sd. $=126 \mu \varepsilon)$, tensile strength $=3,090 \mathrm{MPa}(\mathrm{sd}$. $=38 \mathrm{MPa})$ and elastic modulus $=201.4 \mathrm{GPa}(\mathrm{sd}$. $=$ $29 \mathrm{GPa})$.

Several linear variable differential transformers (LVDTs) were used as shown in Figure 5. The slip at the free end of the bonded FRP is in turn calculated from the difference between LVDT 1 and the average of LVDTs 2 and 3 in addition to consideration of the elastic deformation of the unbonded region of FRP and concrete between. Several electric resistance strain gauges were also installed on the surfaces of the strengthening plates however such strain gauge results are not reported in this paper. Load was applied monotonically by displacing the ram of the universal testing machine shown in Figure 1 at a constant rate of $0.3 \mathrm{~mm} / \mathrm{min}$.
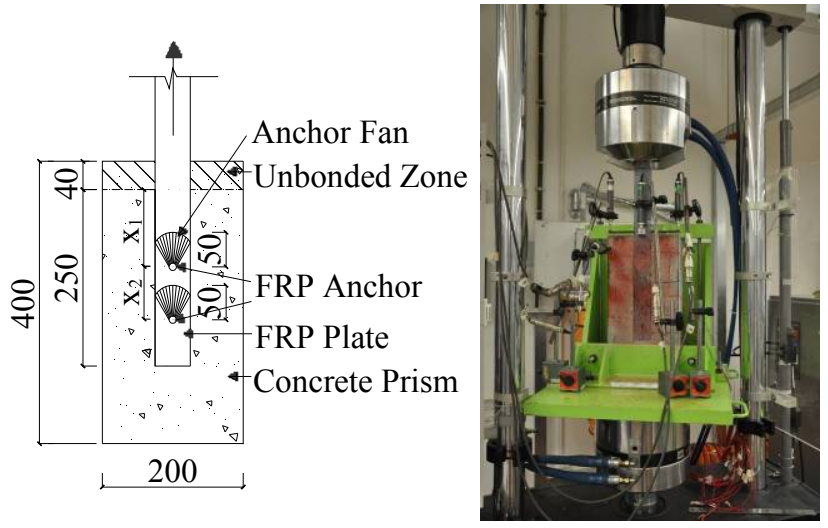

Figure 1. Test set-up.

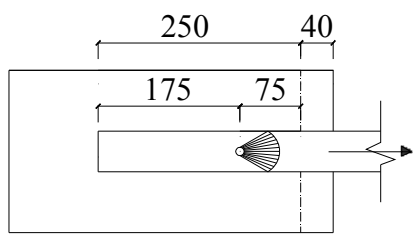

(a) Specimen $1 \mathrm{~A}-3$

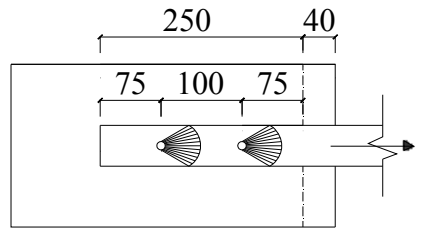

(c) Specimens 2A100-1 2

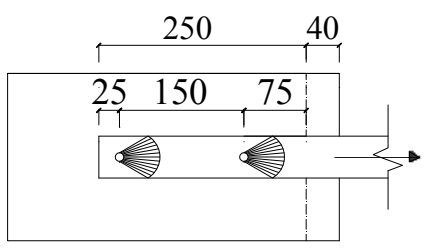

(e) Specimens 2A150-1 2

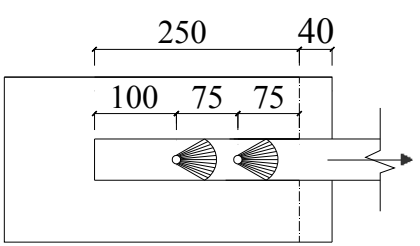

(b) Specimens 2A75-1 2

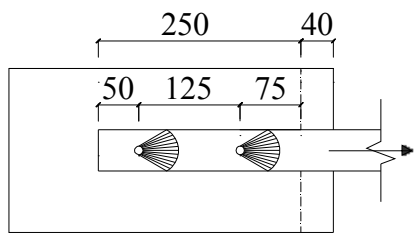

(d) Specimens 2A125-1 2

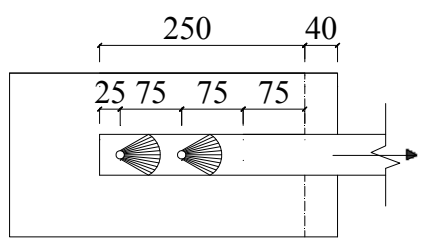

(e) Specimens 2A75a-1 2
Figure 2. Anchor layout (e.g. 2A100-1 2 represents specimens 1 and 2 for joints with 2 anchors spaced at $100 \mathrm{~mm}$ ).

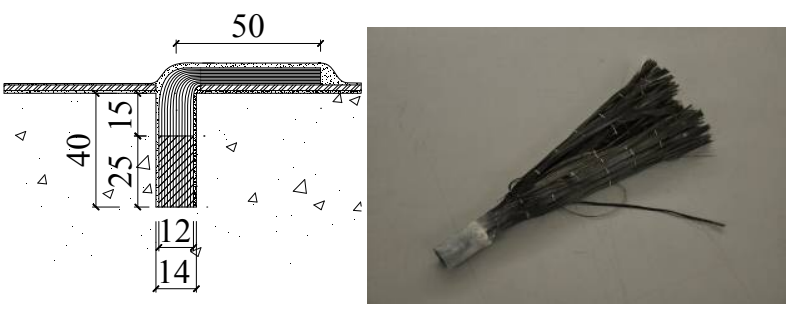

Figure 3. Impregnated carbon FRP anchor details.

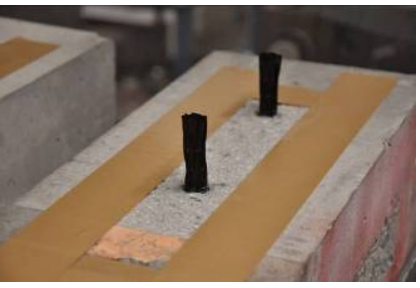

(a) Installation of anchors

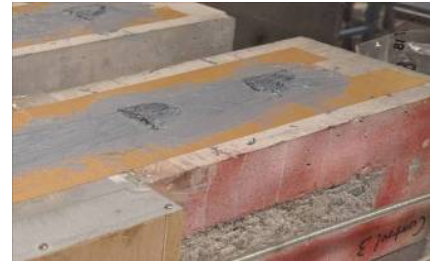

(b) Installaton of FRP plate
Figure 4. Construction of test specimens.

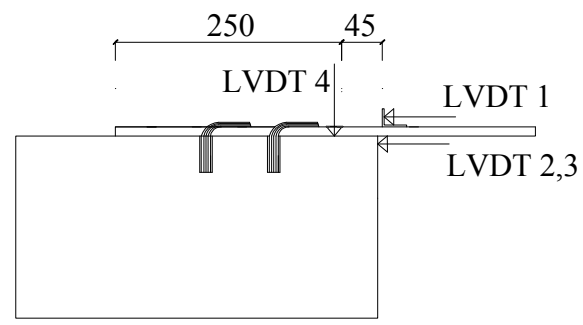

Figure 5. Instrumentation.

\section{TEST RESULTS}

The following is a brief summary and discussion of the results. A much more detailed account is presented in Zhang and Smith (2010).

\subsection{Strength and effect of anchor position}

A summary of the average increase in joint strength for each anchor configuration, relative to the average of the unanchored control joints, is provided in Figure $6 \mathrm{a}$. The increase in joint strength relative to the single anchored joint strength is provided in Figure $6 \mathrm{~b}$. The average strength of the unanchored control joints is $18.0 \mathrm{kN}$ and the strength of the single anchored control joints is $31.1 \mathrm{kN}$.

The effect of the different anchor positions is evident in Figure 6 in which the effectiveness of the second anchor decreases as it is positioned further away from the loaded end. It is currently not clearly understood why the strength of specimen 2A150 dropped below that of the average of the single anchored control joint.

\subsection{Failure Modes}

The three unanchored joints failed by debonding which initiated at the loaded end of the joint. Debonding occurred at the interface of the FRP and the concrete with a thin layer of concrete remaining 
attached to the surface of the FRP (Figure 7a). This desirable mode of failure has been reported numerous times in the literature (e.g. Yao et al. 2005).

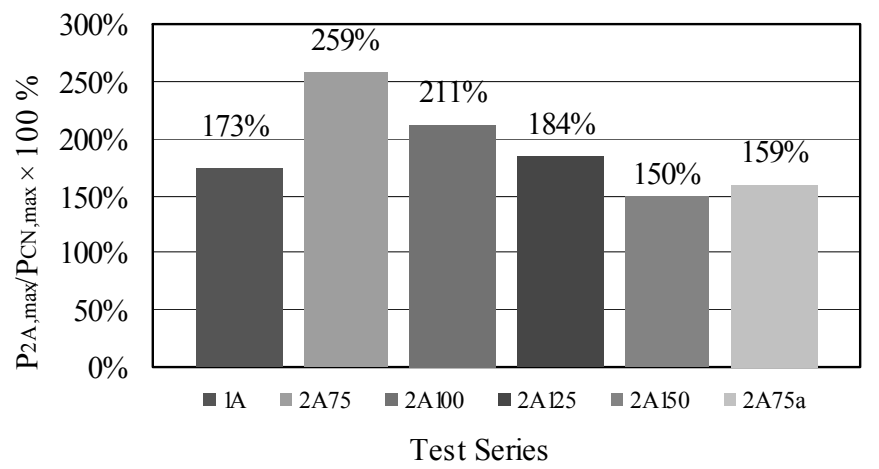

(a) Relative to unanchored control joint average result

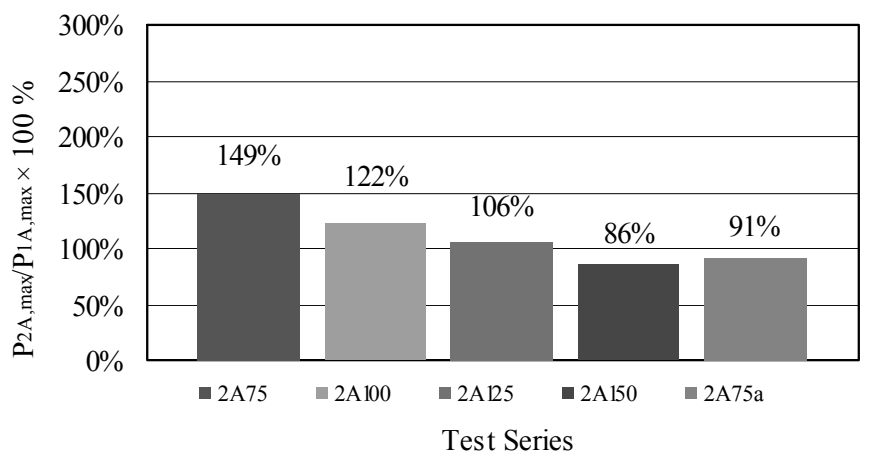

(b) Relative to single anchored control joint average result

Figure 6. Strength enhancement of multiple anchored joints.

The single anchored joints failed by debonding of the plate followed by rupture of the anchor fibres in the bend region. Plate debonding initiated at the loaded end of the joint and the anchor failed after significant slip occurred between the FRP plate and concrete substrate.

For all joints anchored with multiple FRP anchors, the plate completely debonded and then the remaining anchored plate failed in different modes. The following description provides a more detailed account for the majority of the tests.

1. Plate debonding initiated at approximately the capacity of the unanchored control joints. This is particularly evident in the load-slip responses presented in the following sub-section. Such debonding initiated at the loaded end of the plate and generally extended to the anchor fan region of the first anchor (i.e. the anchor nearer to the loaded end of the joint).

2. After the debonding crack propagated to the anchor fan region of the second anchor, the curved edge of the first anchor fan cracked but not in all cases.

3. The remaining bonded portion of the plate then debonded upon which a noticeable drop in the load carrying capacity of the joint was noticed. Most joints then experienced a reserve of strength (and slippage) primarily due to shear resistance provided by sliding of the roughened debonded FRP-to-concrete interface. Such shear resistance was assisted by clamping of the FRP plates by the FRP anchors. In most cases, this reserve of strength was less than the load to cause complete plate debonding, however, not always; as observed in the case of specimens 2A125-1 2 (in the following sub-section). In some cases, longitudinal splitting was observed which originated at the anchor dowel.

4. The joints eventually failed by rupture of the FRP plate with debonding of the anchor fan (Figure $7 b$ ) closet to the loaded end or otherwise by rupture of the anchor fibres in the same anchor (Figure 7c). In most cases, the anchor located furthest away from the loaded end was relatively undamaged but not always.

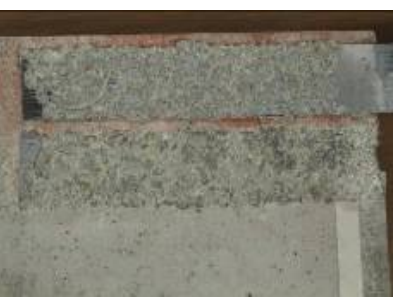

(a) Debonded plate

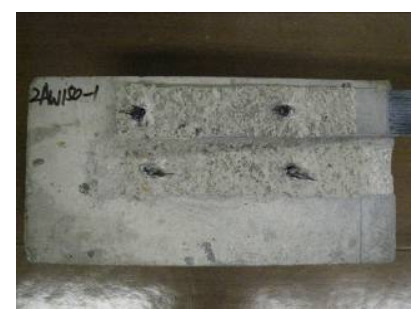

(c) Anchor rupture

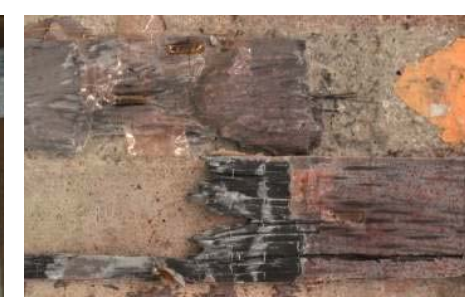

(b) Plate rupture and fan debond
Figure 7. Typical FRP anchor and plate failures.

\subsection{Load-slip responses}

The load-slip responses for all specimens are shown in Figure 8. On account of the long bond length (about double the effective bond length), the control joints exhibited a peak load plateau (Figure 8a). For all of the anchored joints, the peak load at approximately 1 to $2 \mathrm{~mm}$ of slip represents complete debonding of the plate. After debonding, the load generally reduced or remained about the same. In most cases, the multiple anchored joints enjoyed strengths larger than the single anchored joints. Even though the slip capacity of the multiple anchored joints was not in excess of the single anchored joints, the deformability of the former was established. 


\section{CONCLUSIONS}

The results of a series of tests on FRP-to-concrete joints which have been anchored with multiple anchors have been presented in this paper. In most cases, the strength of the joint was increased above that of the single anchored joint. Load-slip responses have enabled the post-strength reserve of strength offered by the anchors after complete debonding of the strengthening plate to be observed and quantified and the significant slip capacity of the anchored joints to be established.

\section{ACKNOWLEDGEMENTS}

Funding provided by the Hong Kong Research Grants Council's General Research Fund Grant HKU 715907E is gratefully acknowledged.

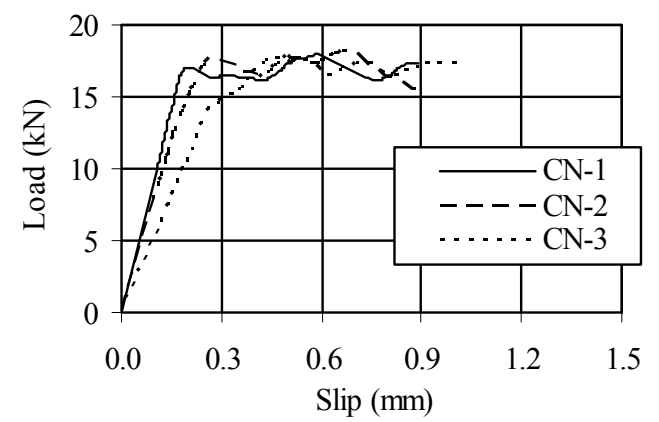

(a) Control joints

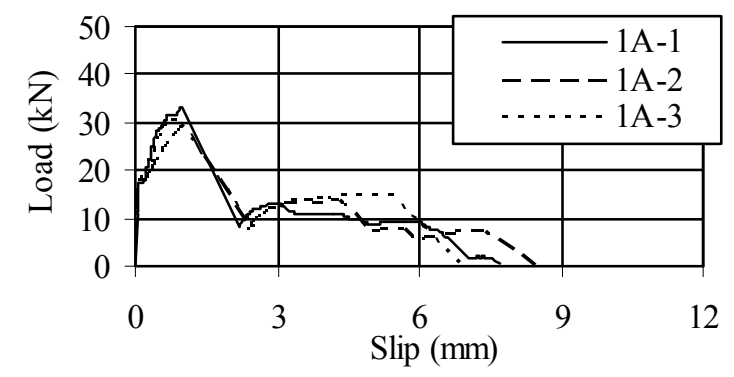

(b) Single anchored joint

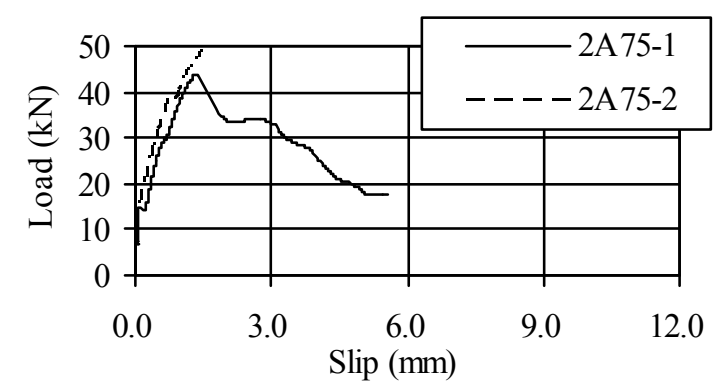

(c) Double anchored joints: spacing $=75 \mathrm{~mm}$

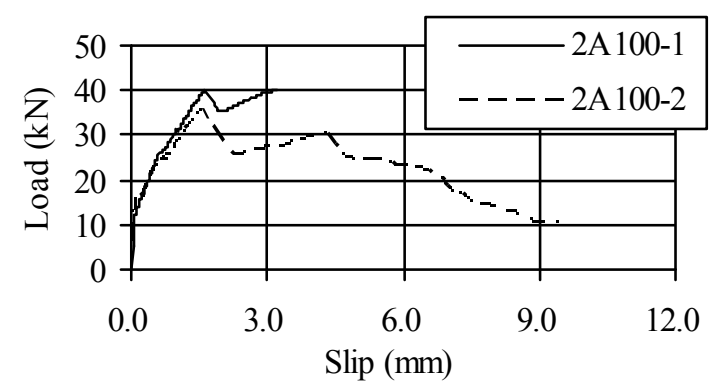

(d) Double anchored joints: spacing $=100 \mathrm{~mm}$

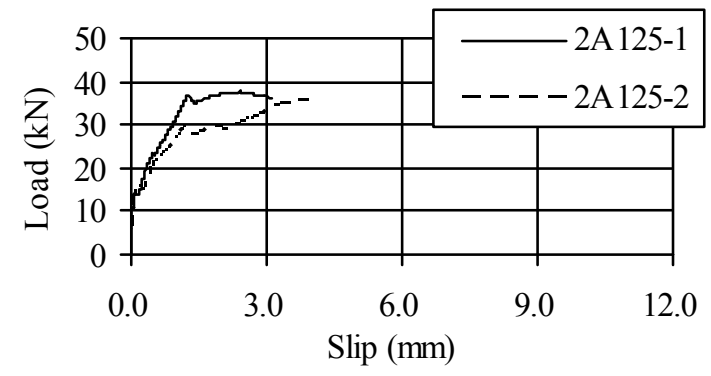

(e) Double anchored joints: spacing $=125 \mathrm{~mm}$

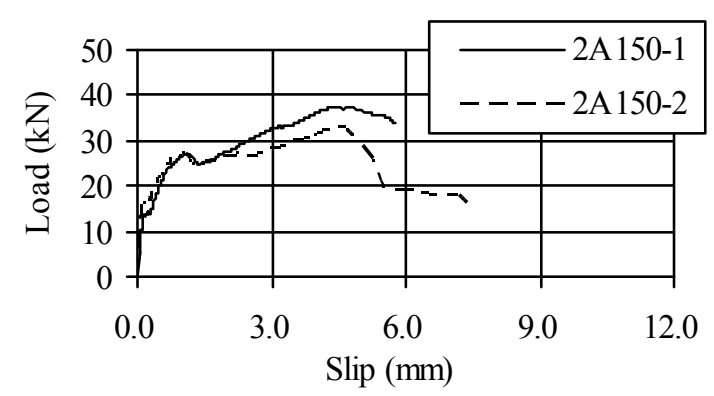

(f) Double anchored joints: spacing $=150 \mathrm{~mm}$

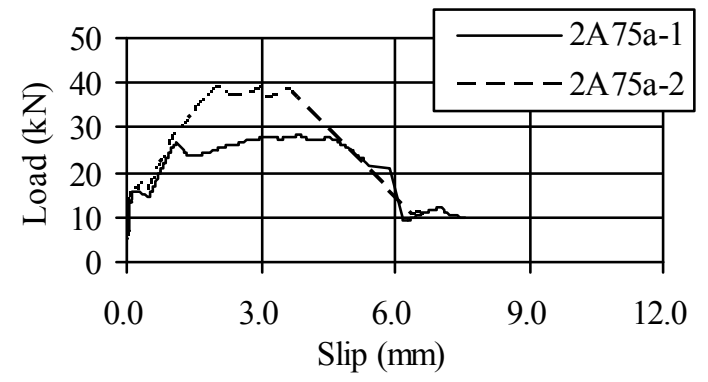

(g) Double anchored joints: spacing $=75 \mathrm{~mm}$

Figure 8. Load-slip responses.

\section{REFERENCES}

Hollaway, LC \& Teng, JG 2008. Strengthening and rehabilitation of civil infrastructures using fibrereinforced polymer (FRP) composites, Woodhead Publishing, UK.

Smith, ST 2009. FRP anchors: recent advances in research and understanding, Second Asia-Pacific Conference on FRP in Structures, APFIS 2009, Korea, 911 December, pp. 35-44.

Yao, J, Teng, JG \& Chen, J. 2005. Experimental study on FRP-to-concrete bonded joints, Composites: Part B, $36,99-110$.

Zhang, HW, Smith, ST \& Kim, SJ 2010. Optimisation of carbon and glass FRP anchor design, under review. 
Zhang, HW \& Smith, ST 2010. Strength and behaviour of FRP anchor groups in FRP-to-concrete joints, in preparation. 